\title{
Crystalline Penicillin for Community Acquired Pneumonia: Does it still work?
}

Nirjala Aryal', Arun Kumar Neopane ${ }^{1}$, Moon Thapa ${ }^{1}$, Umesh Kumar Singh ${ }^{1}$, Keshav Agrawal .

${ }^{1}$ Department of Paediatrics, Shree Birendra Hospital, Chhauni, Kathmandu, Nepal.

\begin{abstract}
Introduction: Pneumonia is the most common cause of mortality and morbidity in children in underdeveloped countries. The common bacterial agents are Streptococcus pneumonia followed by Haemophilus influenzae type b. The only measure to treat bacterial pneumonia is the correct use of antibiotics along with oxygen in moderate to severe cases. The objectives of this study were to see the clinical features of community-acquired pneumonia and to observe the response to treatment with crystalline penicillin in hospitalized children. Methods: This study was a prospective study. The children aged between two months to 59 months with pneumonia were treated with intravenous crystalline penicillin. Response was observed by normalization of respiratory rate and absence of lower chest indrawing. Results: Out of 88 children treated, 79(89.8\%) showed improvement in 48 hours. In children who had tachypnoea, $62.9 \%$ showed normalization in respiratory rate in the first 24 hours and 37.1 percent in 48 hours of treatment. Similarly, among children with lower chest indrawing; $61.1 \%$ showed improvement in 24 hours and the remaining in 48 hours. In 24 hours of treatment $17.7 \%$ of children became afebrile and $46.8 \%$ in 48 hours of treatment. Conclusion: The most common clinical features like cough, fever, tachypnoea and lower chest indrawing can be used to diagnose CAP where chest X-ray is not possible. The response to treatment with crystalline penicillin is very good and, thus, can be used as the first line drug in the treatment of children with CAP.
\end{abstract}

Keywords: community acquired pneumonia, crystalline penicillin, tachypnoea, hypoxia.

\section{INTRODUCTION}

Community-acquired pneumonia (CAP) is defined as an acute infection of the pulmonary parenchyma in a patient who has acquired the infection in the community ${ }^{1}$. It is caused by a number of infectious agents, including viruses, bacteria and fungi. The most common bacteria in causing pneumonia in children are Streptococcus pneumoniae followed by Haemophilus influenzae type b $(\mathrm{Hib})^{2,3}$.

CAP is the most common cause of childhood deaths in the developing countries ${ }^{4}$. In the developed countries the burden of the disease is in order of $10-15$ cases $/ 1000$ children per year and a hospital admission rate of 1-4/1000 per year ${ }^{5}$. Use of antibiotics is one of the main strategies used to overcome children's morbidity and mortality in such circumstances ${ }^{6}$. World Health Organization (WHO) has recommended penicillin $G$ to children hospitalized with severe CAP in developing countries ${ }^{7,8}$. The rational for such a choice is, to treat Streptococcus Pneumonia, which is the most common cause of bacterial CAP who are appropriately treated could be seen clinically within 24 to 48 hours 9 . Penicillin resistant strains of streptococcus pneumonie is emerging worldwide ${ }^{10,11}$. Intermediate or high-level resistance to penicillin has become a significant problem. Children, particularly those living in child care facilities and those receiving frequent courses of antibiotics, appear to be important carriers of resistant strains ${ }^{12}$. Thus the objective of this study was to observe the clinical response of the children hospitalized with communityacquired pneumonia to the treatment with crystalline penicillin and to see the clinical features of communityacquired pneumonia in hospitalized children.

\section{METHODS}

\section{Correspondence:}

Maj. Dr. Nirjala Aryal

Department of Paediatrics, Shree Birendra Hospital.

Kathmandu, Nepal

Email: nirjalaaryal@gmail.com

Phone: 
This was a prospective study conducted from $30^{\text {th }}$ January 2011 to $1^{\text {st }}$ January 2012. After obtaining informed consent from the parents or caretakers, children aged between two months to 59 months with fever (axillary temperature $\geq$ $38^{\circ} \mathrm{C}$ ), fast breathing (defined as respiratory rate $\geq 50 / \mathrm{min}$ in 2-11 months and $\geq 40 / \mathrm{min}$ in 12-59 month aged child) and/or with difficulty in breathing (defined by bilateral lower chest wall indrawing) ${ }^{10}$ and children with chest $\mathrm{X}$-ray findings suggestive of pneumonia were included in this study.

Among the enrolled children the respiratory rate and chest indrawing were observed when the children were calm and quiet. Besides, Oxygen saturation $\left(\mathrm{SpO}_{2}\right)$ was monitored using pulse oximeter with a finger probe. The respiratory rate was counted twice if it was equal or above the reference range for each age group. The second count was recorded as the RR for the child. The RR count was done by experienced paediatrician. Hypoxemia was defined as oxygen saturation less than $90 \%$ in room air ${ }^{10}$. Pneumonia was confirmed if a pulmonary infiltrate or pleural effusion was described by a qualified radiologist.

Fever was treated with paracetamol as and when required and hypoxemia if present was treated with oxygen via nasal cannula. Those children that qualified the above criteria were hospitalized and treated with intravenous crystalline penicillin (CP) @200,000 IU/kg/day in four divided doses after the skin sensitivity test. Axillary temperature, respiratory rate, chest indrawing and oxygen saturation of the enrolled children were recorded 6 hourly. The response was measured by normalization of respiratory rate and absence of chest indrawing at 24 hours and 48 hours of treatment. If no improvement were seen in 48 hours of intravenous $\mathrm{CP}$, the child were treated with other antibiotics as per the hospital protocol. Improvement in signs and symptoms were considered "improved" only at 24 hour and 48 hours of initiation of treatment to allow adequate time for action of antibiotics.

Children with underlying debilitating or chronic pulmonary illnesses and heart disease, children already taking oral antibiotics at the time of enrollment, those who are known allergic to penicillin group of drugs, patients requiring referrals to other centers for various reasons and children without evidence of pneumonia on chest X-Ray were excluded from this study.
Figure 1. treatment outcome of patients.

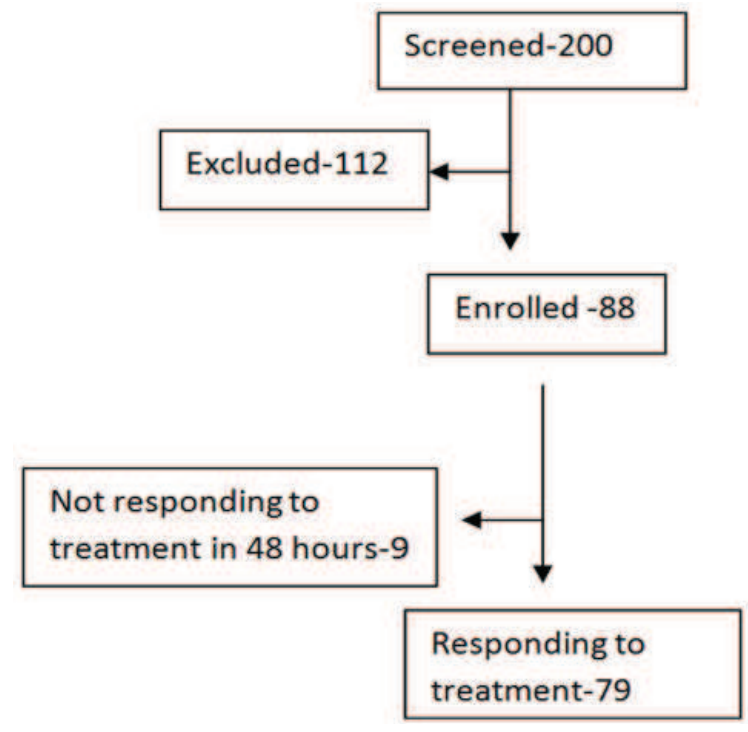

RESULTS

A total of 200 children were screened and 88 children who met the inclusion criteria were enrolled in the study. Among the enrolled patients $55.68 \%$ were males. The mean age of children was 33.26 in the age group of 2-59 months. There were 14(15.9\%) children within the age group 2-12 months and $74(84.1 \%)$ children aged more than $12-59$ months.

Table1. Showing improvement in signs and symptoms after treatment with $\mathrm{CP}$.

\begin{tabular}{|lcc|}
\hline Signs and Symptoms & $\begin{array}{c}\text { Improvement } \\
\text { after 24 hrs }\end{array}$ & $\begin{array}{c}\text { Improvement } \\
\text { after 48 hrs }\end{array}$ \\
\hline $\begin{array}{l}\text { Tachypnea } \\
\text { Lower chest indrawing } \\
\text { and tachypnea }\end{array}$ & $15(57.7 \%)$ & $24(38.7 \%)$ \\
$\begin{array}{l}\text { Fever } \\
\begin{array}{l}\text { Decrease in Cough as re- } \\
\text { ported by mother }\end{array}\end{array}$ & $\begin{array}{l}\text { 14(17.7\%) } \\
\text { Hypoxia }\end{array}$ & $37(46.8 \%)$ \\
\hline
\end{tabular}

Out of 88 children treated with CP, 79(89.8\%) responded well. In $62(70 \%)$ children who presented with tachypnoea $53.2 \%$ had normalization of respiratory rate in the first 24 hours of intravenous CP and $38.7 \%$ of the children had normalization of respiratory rate within 48 hours of treatment. Similarly out of $26(29.5 \%)$ children who had lower chest indrawing and tachypnoea at the time of enrollment $57.7 \%$ of the children showed disappearance of lower chest indrawing and normalization of respiratory rate in first 24 hours and $26.9 \%$ children in 48 hours of treatment. None of the enrolled children had lower chest indrawing in isolation without tachypnoea. 


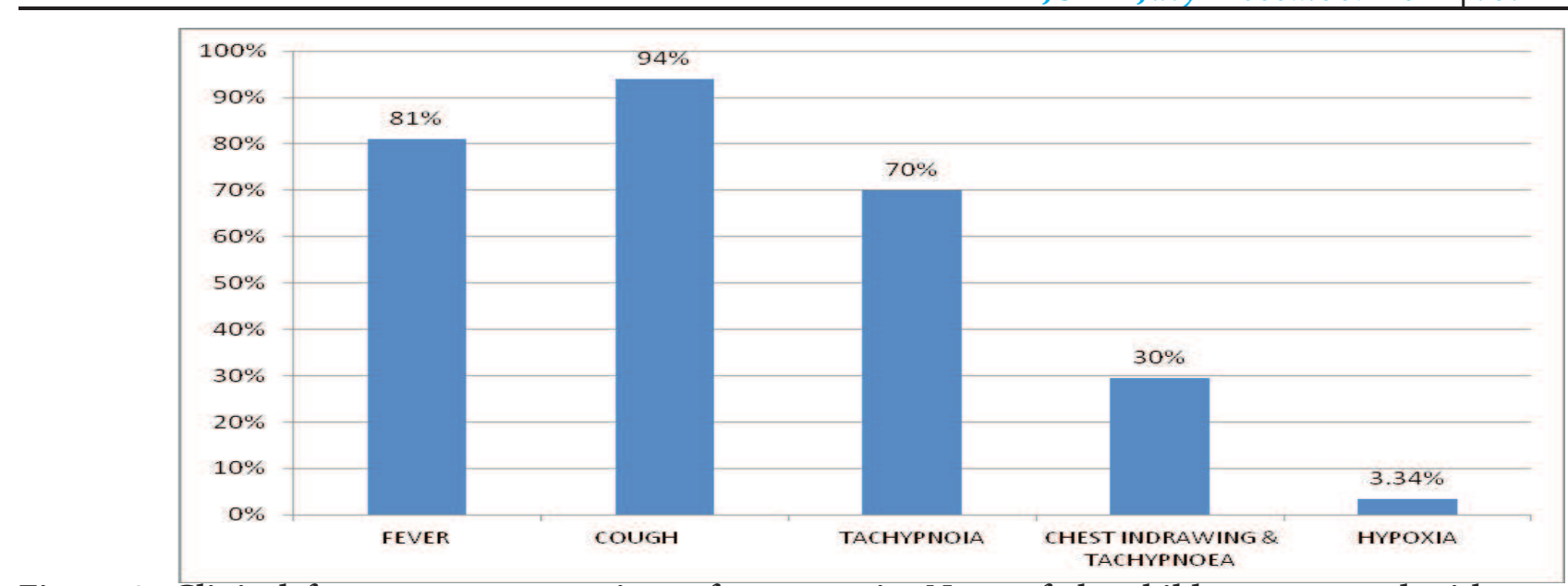

Figure 2. Clinical features at presentation. of pneumonia. None of the children presented with cyanosis and dehydration.

All of the three (3.34\%) children presenting with hypoxia at the time of enrollment maintained their Sp02 above $90 \%$ at room air in the first 24 hours of treatment with intravenous CP. Among children who presented with fever, $17.7 \%$ became afebrile in 24 hours and $46.8 \%$ became afebrile in 48 hours of treatment and after that there was no need of Paracetamol in them. Out of $83(94 \%)$ children presenting with cough, the mother reported decrease in cough only in $6 \%$ of children in 24 hours and $21.7 \%$ in 48 hours of treatment.

All the enrolled case recovered completely. Out of 9 cases who didn't respond to CP , two developed pleural effusion, one empyema thoracic and six remained tachypnoeic with chest indrawing even after 48 hours of CP.

\section{DISCUSSION}

The estimated incidences of pneumonia in India, Pakistan and Bangladesh are 44 million, 7 million and 6 million repectively ${ }^{2}$. The demographic and health survey done in Nepal in 2011 showed that $5 \%$ of the children less than five years of age had symptoms of acute respiratory illness (ARI), 19\% had fever and 14\% had diarrhea 2 weeks preceding the survey. ARI and severe diarrhoea causing dehydration are the major causes of childhood mortality in $\mathrm{Nepal}^{3}$. But the published data of death due to pneumonia in children less than five years of age is lacking.

In our study the main presenting clinical feature of pneumonia was cough (94\%) followed by fever, tachypnoea and tachypnoea with lower chest indrawing. The least common feature was hypoxia. This is supported by a similar study done in Himachal Pradesh, India where the most common presenting complaints were fever and cough followed by rapid or difficulty in breathing. ${ }^{13}$
Similarly a study done in children $>1$ year of age with the first episode of wheezing found that the combination of tachypnea, tachycardia, fever, and localized findings (rales or wheezing) both before and after bronchodilator therapy could identify $95 \%$ of patients with pneumonia. ${ }^{14}$ Another study done in 154 hospitalized children aged more than two months with CAP showed that the most common presenting complaints of pneumonia were cough (99.2\%), fever (97.2\%) and difficulty in breathing (56.5\%). The findings were tachypnea (75.2\%), fever (49.7\%) and crackles $(33.8 \%) \cdot{ }^{15}$ All these show that fever, cough and tachypnoea can be used as the diagnostic tool for pneumonia where chest X-ray is not always possible especially in rural and under equipped health settings.

Since most of the causative agents of childhood pneumonia cannot be detected, antibiotic treatment is most often empiric, especially in underdeveloped countries. Various antibiotics are being used in the treatment protocol of CAP worldwide ${ }^{16}$ and so also in Nepal. In our study the data showed that CP successfully treated the great majority $(89.8 \%)$ of the children aged between 2 to 59 months with radiographically confirmed CAP. These results are also similar to the result shown in the retrospective cohort study done in hospitalized children with CAP in Brazil where Penicillin G successfully treated $82 \%(126 / 154)$ of the study group and the improvement was markedly seen on the first day of treatment itself. ${ }^{15}$ Another study done in Finland showed that out of 153 children hospitalized for uncomplicated CAP, $66 \%$ were treated with penicillin $\mathrm{G}$ and they also showed a rapid and uneventful recovery. ${ }^{17}$

Penicillin G is still considered a drug of choice in hospitalized children with CAP even in many European countries with low penicillin resistance of pneumococci. ${ }^{18,19,20}$ Penicillin G is no longer recommended in the United States as the first- 
choice drug because of limited supply and the increasing resistance of pneumococci to penicillin ${ }^{21}$, whereas in western countries like Finland, 95\% of pneumococcal strains still remained sensitive to penicillin. ${ }^{22}$ Since a majority of children with CAP responded significantly well to CP in our study, it could still be considered a drug of choice in hospitalized children with CAP in low income and resource poor countries like Nepal.

Since the aim of the study was to see the response to treatment with antibiotics $(\mathrm{CP})$ in diagnosed cases of pneumonia, the onset of symptoms of ARI, days of hospital admission, nutritional status and other confounding variables were not included in this study. This was a descriptive study in which a cohort of children was followed up. Therefore, further statistical analysis was not considered of additional value. The relatively small sample size was also one of the limitations of this study.

\section{CONCLUSIONS}

Crystalline Penicillin is a very good drug for the treatment of CAP and can still be used as the first drug in the treatment of children with CAP. The most common clinical features like cough, fever, tachypnoea and lower chest indrawing can still be used in the diagnosis of CAP where chest Xray facilities are absent. This study however had certain limitations like; absence of bacteriological diagnosis and a relatively small sample size.

\section{ACKNOWLEDGEMENT}

The authors would like to thank all the staffs of the Department of Paediatrics at Shree Birendra Hospital We are also very grateful to the consultant doctors of the department of radiology without whom the study would not have been been completed.

\section{REFERENCES}

1. Theodore C.Sectish, Charles G. Prober. Pneumonia. In: Kliegman, Behrman, ST. Geme, Schor, Stanton, editors. Nelson Text book of pediatrics. 19th ed. Philadelphia: Saunder Elsevier; 2012: p.1474-9.

2. World Health Organization. Pneumonia: The forgotten killer of children. The United Nations Children's Fund /World Health Organization; Sept 2006.

3. Ministry of health and population, New Era, ICF Micro, USAID. Nepal Demographic and Health Survey 2011. Katmandu: Ministry of health and population, government of Nepal; 2011.

4. Mulholland K. Childhood pneumonia mortality-a permanent global emergency. Lancet 2007;370:285-9.

5. Farha T, Thomson AH. The burden of pneumonia in children in the developed world. Paediatr Respir Rev 2005;6:76-82.

6. Sazawal S, Black RE. Effect of pneumonia case management on mortality in neonates, infants, and preschool children: a meta-analysis of community-based trials. Lancet Infect Dis 2003;3:547-56.

7. World Health Organization. A manual for doctors and other senior health workers. Programme for the Control of ARI. Geneva: WHO; 1990. ARI in children: case management in small hospitals in developing countries.

8. Addo-Yobo E, Chisaka N, Hassan M, Hibberd P, Lozano JM, Jeena $P$, et al. A randomized multicentre equivalency study of oral amoxicillin versus injectable penicillin in children aged 3 to 59 months with severe pneumonia. Lancet 2004;364:1141-8.

9. Sandora TJ, Harper MB. Pneumonia in hospitalized children. Pediatr Clin North Am 2005; 52:1059.

10. Thornsberry C, Ogilvie PT, Holley HP Jr, Sahm DF. Survey of susceptibilities of Streptococcus pneumoniae, Haemophilus influenzae, and Moraxella catarrhalis isolates to 26 antimicrobial agents: a prospective U.S. study. Antimicrob Agents Chemother 1999; 43:2612.

11. Raymond J, Le Thomas I, Moulin F, et al. Sequential colonization by Streptococcus pneumoniae of healthy children living in an orphanage. J Infect Dis 2000;181:1983.

12. Sisson BA, Buck G, Franco SM, et al. Penicillin minimum inhibitory concentration drift in identical sequential Streptococcus pneumoniae isolates from colonized healthy infants. Clin Infect Dis 2000;30:191.

13. Bhavneet B, Sahul B, Vandna V. Role of Acute Illness Observation Scale (AIOS) in Managing Severe Childhood Pneumonia. Indian J Pediatr 2007;74:27.

14. Gershel JC, Goldman HS, Stein RE, et al. The usefulness of chest radiographs in first asthma attacks. N Engl J Med.1983;309(6):336-39. 15. Simbalista R, Araújo M, Nascimento-Carvalho, C.Outcome of children hospitalized with community-acquired pneumonia treated with aqueous penicillin G. Clinics (Sao Paulo). 2011;66(1):95-100.

16. Feyzullah C, Abdulkadir G, Gunsel K. Comparison of Two Antibiotic Regimens in the Empirical Treatment of Severe Childhood Pneumonia. Indian J Pediatr 2004;71(11):969-72.

17. Juvén T, Mertsola J, Waris M, Leinonen M, Ruuskanen O. Clinical response to antibiotic therapy for community-acquired pneumonia. Eur J Pediatr 2004;163:140-4.

18. Hedlund J, Ortqvist A. Management of patients with communityacquired pneumonia treated in hospital in Sweden. Scand J Infect Dis 2002;34:887-89

19. Moroney JF, Fiore AE, Harrison LH, Patterson JE, Farley MM, Jorgensen $J H$ et al. Clinical outcomes of bacteremic pneumococcal pneumonia in the era of antibiotic resistance. Clin Infect Dis 2001;33:797-805

20. Ruuskanen O, Mertsola J. Childhood community acquired pneumonia. Semin Respir Infect 1999;14:163-72

21. Nelson JD. Community-acquired pneumonia in children: guidelines for treatment. Pediatr Infect Dis J 2000;19:251-53

22. Pihlajamaki M, Kotilainen P, Kaurila T, Klaukka T, Palva E, Huovinen P. Macrolide-resistant Streptococcus pneumonia and use of antimicrobial agents. Clin Infect Dis 2001;33:483-88 